\title{
The impact of auditory distraction on retrieval of visual memories
}

\author{
Peter E. Wais • Adam Gazzaley
}

Published online: 22 September 2011

(C) Psychonomic Society, Inc. 2011

\begin{abstract}
Recent research has revealed that the presence of irrelevant visual information during retrieval of long-term memories diminishes recollection of task-relevant visual details. Here, we explored the impact of irrelevant auditory information on remembering task-relevant visual details by probing recall of the same previously viewed images while participants were in complete silence, exposed to white noise, or exposed to ambient sounds recorded at a busy café. The presence of auditory distraction diminished objective recollection of goal-relevant details, relative to the silence and white noise conditions. Critically, a comparison with results from a previous study using visual distractors showed equivalent effects for auditory and visual distraction. These findings suggest that disruption of recollection by external stimuli is a domain-general phenomenon produced by interference between resourcelimited, top-down mechanisms that guide the selection of mnemonic details and control processes that mediate our interactions with external distractors.
\end{abstract}

Keywords Episodic retrieval $\cdot$ Interference $\cdot$ Domain general $\cdot$ Cognitive neuroscience of memory Episodic memory $\cdot$ Cognitive neuroscience of perception and attention

P. E. Wais $(\bowtie) \cdot$ A. Gazzaley $(\bowtie)$

Departments of Neurology, Physiology and Psychiatry,

W. M. Keck Center for Integrative Neurosciences,

University of California,

UCSF - MC2512, 1600 16th St, Room N474,

San Francisco, CA 94158-2330, USA

e-mail: peter.wais@ucsf.edu

e-mail: adam.gazzaley@ucsf.edu

\section{Introduction}

When trying to remember detailed information about prior experiences, we engage internal selection processes to focus cognitive resources on the retrieval effort (Atkinson \& Juola, 1973; James, 1890; Mandler, 1980). Accordingly, environmental stimulation that inundates our senses with information irrelevant to our memory goals can distract us during recall. Specifically, recent research from our laboratory documented an impact of irrelevant visual information on recognition such that visual distraction diminishes retrieval of specific details relevant to current memory goals (Wais, Rubens, Boccanfuso, \& Gazzaley, 2010). Whether the influence of irrelevant auditory information impacts objective recollection of visual details has not been examined. The goal of the present study was to examine the effect of auditory distraction on retrieval of episodic memory and, taking into account our previous findings, the possibility that environmental distraction may induce domain-general interference that disrupts recollection.

Wais et al. (2010) used a novel paradigm to manipulate visual distraction during retrieval of long-term memory (LTM) while recording with functional MRI (fMRI). The participants' recall for a particular visual detail from previously studied images (i.e., the number of objects) was probed with auditory cues in separate blocks when their eyes were closed, their eyes were open with gaze fixed on a gray screen, and their eyes were open with gaze fixed on a complex visual scene. Critically, participants were instructed to direct their full attention to the memory retrieval task and had no goals to attend to distracting stimuli. The study revealed that recollection of goalrelevant visual details was diminished in the presence of 
irrelevant visual information, whereas item recognition of the studied objects was not impacted (Wais et al., 2010).

Episodic memory is thought to reconstruct specific ensembles of mnemonic details (Moscovitch, 2000) and can involve mental imagery mechanisms to retrieve internal representations of perceptual information from past events (Ishai, Ungerleider, \& Haxby, 2000; Kosslyn, Ganis, \& Thompson, 2001; Tulving, 1985). Neural evidence based on fMRI data supports this interpretation by showing that recollection involves the reinstatement of activity in visual cortices associated with the encoding of a prior event (Johnson, McDuff, Rugg, \& Norman, 2009; Johnson \& Rugg, 2007; Wheeler \& Buckner, 2004). In the context of recent data that show that reconstruction of detailed memory is disrupted by the influence of irrelevant external information (Wais et al., 2010; Wais, Kim, \& Gazzaley, 2011), an interesting and unanswered question is whether diminished performance is the result of interference at sensory-modality-specific stages of representational processing or, more generally, at processes involved in the selection of goal-relevant details retrieved from LTM (i.e., mnemonic selection).

The present study utilized an experimental paradigm that paralleled the previous study but substituted auditory in place of visual distractors. Specifically, written cues probed recall of visual details of previously studied objects when participants were (1) in complete silence, (2) exposed to white noise, or (3) exposed to ambient sounds recorded at a busy café (see Fig. 1). Critically, the target stimuli and encoding procedure in the present study with auditory distraction were identical to those in the previous study with visual distraction. However, unlike in the previous study, in which visual distraction competed for visual processing resources involved in mental imagery, in the present study, bottom-up processing of auditory stimuli and processing of internal visual representations of items in memory were largely supported by discrete sensory cortices. We hypothesized that a comparison of effects of external distraction from different sensory modalities, therefore, might yield evidence for or against domaingeneral interference on recollection. If auditory distraction effects were present in the present study, the convergence of the results with those from the prior study would suggest that external interference effects on retrieval occur at the level of domain-general processes, such as an episodic buffer (Baddeley, 2010) or mnemonic selection (Anderson \& Spellman, 1995). Alternatively, the disruption of recollection of goal-relevant visual details by visual distractors, but not by auditory distractors, would suggest that retrieval of LTM involving visual imagery is susceptible to intramodality interference at the visuospatial sketchpad (Baddeley, 2010). By exploring distraction effects across sensory modalities, we can better understand processes that are critically involved in how we reconstruct the past while navigating the external environment.

Research examining the effects of irrelevant background sound on tasks involving working memory (WM) maintenance has revealed a negative impact on immediate serial recall (Beaman, 2005; Campbell, Beaman, \& Berry, 2002; Tremblay, Nicholls, Alford, \& Jones, 2000), as well as significant changes in neural activity associated with concurrent task-relevant visual processing (Weisz \& Schlittmeier, 2006). The irrelevant sound effect (ISE) is the decline in accuracy for immediate memory of serial order when the test is completed in the presence of irrelevant sound, relative to complete silence. Although the ISE is robust for human speech (Campbell et al., 2002; Salame \& Baddeley, 1982), behavioral and neural results do not show a disruptive effect on immediate serial recall from less descriptive background noise (Little, Martin, \& Thomson,
Fig. 1 Experimental paradigm. A schematic of the procedure shows the study session, in which participants answered two incidental questions about each of 168 images ( 3 s per presentation), and the test session, in which visual cues described 168 targets and 36 lures in singular form $(2.5 \mathrm{~s}$ per presentation, 5.5 -s intertrial interval). The visual probes cued participants' recall while they wore headphones during trials presented in three conditions

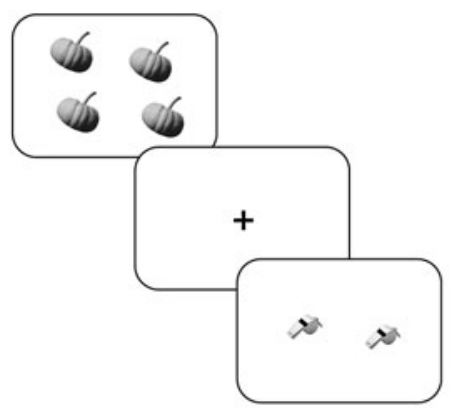

Study session- answer yes or no first presentation: "does one fit inside a lady's shoebox?" second presentation: "can you carry all of them at once?"

Test session- recall and respond 1, 2, 3, 4 or new

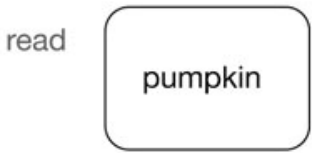


2010; Weisz \& Schlittmeier, 2006). Quite distinct from the effect of irrelevant sound on immediate recall of serial order, the present study explored the influence of auditory distraction on episodic retrieval, using both ambient café sounds and white noise in the experimental paradigm.

\section{Method}

\section{Participants}

Twenty-eight young adults (18 to 29 years old, 19 males), who were native speakers of English recruited from the university community, gave their informed consent to perform the experimental tasks and were compensated for their time. All participants were screened to ensure that they were healthy, had no history of difficulty in hearing or of neurological or psychiatric disease, and were not taking any psychotropic medication. All participants had normal or corrected-to-normal vision. One participant fell asleep during one block of the silence test condition, and his data were excluded from analysis.

\section{Stimuli}

Visual stimuli included 168 target images and 240 singular nouns (describing the objects or similar lures) presented on a computer screen at $768 \times 1,024$ pixel resolution. Auditory stimuli, presented through a set of Sennheiser eH 150 sound-canceling headphones, included one recording with a flat power spectral density (white noise) and 80 recordings of ambient sounds from a busy restaurant dining room. The recordings of white noise and café sounds were equated and normalized for power spectral density in Audacity ${ }^{\circledR} 1.3 .5 \mathrm{~d}$ digital audio editor. Stimuli were presented during the experiment using E-Prime 2.0.

Each target image displayed one to four copies of the same object from a three-dimensional perspective, in color, on a plain white background. The objects were selected from a stimulus set of common items, developed by Bakker, Kirwan, Miller, and Stark (2008), that were controlled for concreteness and ease in namability. The displacement in the viewable area from the objects was held as constant as possible, whereas the actual objects varied in size (i.e., wishbones vs. sofas). The number of target images with each count of objects (one, two, three or four objects) was equated.

\section{Procedure}

The experiment was separated into two sessions: encoding and test. Written instructions were read out loud to each participant by the experimenter before each session, and the participant then completed a brief practice run for each session with the experimenter. During the encoding session, each of the 168 target images was presented for $3,000 \mathrm{~ms}$, in random order, in two runs. When participants viewed each image during the first run, they entered a yes or no answer into the computer keyboard to indicate their judgment about whether one of the objects from the image could fit inside a lady's shoebox and, during the second run, to indicate whether they believed that they could carry all of the objects from the image across the room using only their hands and arms. The incidental encoding tasks promoted in-depth visualization of the targets without specifically referencing numerosity. Each of the 336 trials was preceded by a 2,000-ms fixation cross, and 12-s rest periods occurred after each block of 56 trials.

The retention interval (i.e., the midpoint of the study phase to the midpoint of the test phase) was nominally $60 \mathrm{~min}$. Following this rest period in which participants were asked to refrain from reading, they were instructed about a surprise memory test, which they practiced while wearing a set of sound-canceling headphones that they adjusted to a loud but comfortable volume level. In order to validate that participants had selected an effective headphone volume level, they were asked about their comprehension of the few discernible words included in the practice trials with auditory distraction.

The test session involved viewing the computer screen, where written cues appeared, which referred back to target images from the study session, and listening through headphones in three conditions: silence, white noise, or prerecorded restaurant chatter that served as an auditory distraction (AD). The memory test was divided into six blocks (40 trials in each block, 32 targets presented in a random order with eight lures) presented in one of three pseudorandom orders. The instructions for each trial were to read the written cue (a word written in black lowercase Helvetica font in the center of a white screen), recall the count of the objects on the target image described by the cue, and enter an answer by pressing 1, 2, 3, 4, or "new" on the computer keyboard. Participants were instructed to give recall responses as rapidly as possible without sacrificing accuracy (the trial advanced 2,500 ms after onset of the cue). A fixation cross was subsequently presented as a 5,500-ms intertrial interval. The white noise and AD test blocks were completed with audio input presented through the headphones concurrent with the 2,500-ms presentation of the visual probe, whereas the headphones were turned off during the silence blocks to minimize any perceptible sound.

At the conclusion of the experiments, participants completed a verbal exit interview in which the experimenter inquired about the strategy adopted to solve the recall question. All 27 participants included in the analysis 
reported that they had used visual imagery of the target images on the majority, if not every one, of the test trials.

\section{Analysis}

The impact of auditory distraction on retrieval of LTM was analyzed through comparison of results across conditions (silence, white noise, $\mathrm{AD}$ ) in three categories of responses to the written cues (i.e., memory subtypes): correct, where responses that gave the correct object count corresponding to a previously studied image (i.e., targets) were interpreted as being based on an objective measure of recollection; incorrect, where responses that gave an incorrect object count for a target were interpreted as being based on item recognition, given that the participant indicated recognition of the target when recollection of details relevant to the current task was weak or unavailable (Mandler, 1980); and false alarms, where cues for unstudied objects (i.e., lures) that were erroneously given an object count were classified as false alarms. The trials on which the cue for a target was given a new response were classified as forgotten, and cues for lures that were given a new response were classified as correct rejections.

\section{Results}

\section{Auditory distraction}

The analysis began with a comparison across conditions of the hit rates (i.e., the proportion of targets in each condition that were given a count, whether the count was correct or incorrect), and the false alarm rates (see Table 1). There was no effect of condition on hit rate, $F(2,52)<1$. A main effect on false alarms was evident, such that false recognition was greatest during silence, $F(2,52)=3.88, p<.03$; pair-wise comparisons: silence $>$ white noise, and silence $>\mathrm{AD}$, both $p$ s $<.05$.

Objective recollection of task-relevant details was analyzed across conditions by comparing conditional correct scores that computed the proportion of target responses for which individuals correctly recalled the count given that an item was not forgotten (i.e., $p$ (Correct) $/[(p$ (Correct) $+p$ (Incorrect) $]$.
A repeated measures ANOVA showed a main effect of condition, $F(2,52)=8.15, p<.001$, such that retrieval of relevant visual details during presentation of the auditory distractors declined significantly, relative to silence and white noise (see Fig. 2, auditory distraction; pairwise comparisons: $p<.001$ and $p<.02$, respectively). There was no difference in the pairwise comparison between silence and white noise. These results revealed that auditory distraction, but not white noise, disrupted recollection of task-relevant details.

Surprisingly, the false alarm rate increased during silence. It is possible that completing a 5-min 20-s test period without auditory stimulation (i.e., silence) caused participants to relax their approach to the recall task during those blocks and modestly loosen their decision criterion. We examined this question with a post hoc analysis of memory discrimination indexed by $d^{\prime}$ (Macmillan \& Creelman, 2005). Although false alarms had differed by condition, the post hoc analysis showed mean $d$ 's of $2.08 \pm 0.10$ for silence, $2.25 \pm 0.10$ for white noise, and $2.32 \pm 0.10$ for $\mathrm{AD}$, and only a trend for an effect of condition on $d^{\prime}, F(2,52)=2.73, p<.08$. This result shows that participants retained availability of item memory for target images despite the disruptive impact from auditory distraction on their capability for retrieving episodic details.

\section{Comparison with visual distraction}

A direct comparison between the results of the present study using auditory distractors and those of the previous behavioral study that used visual distractors (Wais et al., 2010) in a parallel paradigm was performed. In the visual distraction experiment, the conditions analogous to the no-distractor (silence), control distractor (white noise), and distractor (AD) conditions in the present study were the eyes shut (shut), eyes open with gray screen (gray), and eyes open with a complex natural scene (VD) conditions, respectively. The visual distractors were vivid scenes including man-made artifacts and urban and natural environments. During the VD condition, one distracting scene was presented on each trial. Of note, in comparison with the VD condition, stimulation from ambient café sounds during the $\mathrm{AD}$ condition is more dynamic than that from static visual images.

The impact on recollection from auditory distraction and visual distraction was compared in a mixed-design, 2
Table 1 Behavioral results: A summary shows statistics for the responses to 168 targets and 36 lures that were presented during the test session. The mean performance for 27 participants is given in each of the three auditory conditions (standard error of the mean)

\begin{tabular}{lllll}
\hline & \multicolumn{2}{l}{ Targets } & & \multicolumn{1}{l}{ Lures } \\
\cline { 2 - 4 } & Correct & Incorrect & hit rate & False alarms \\
\hline Total & $58.0 \%(2.5)$ & $32.2 \%(2.3)$ & $90.2 \%(2.4)$ & $23.9 \%(2.0)$ \\
Silence & $60.9 \%(2.7)$ & $29.9 \%(2.4)$ & $90.8 \%(2.7)$ & $27.3 \%(2.3)$ \\
White Noise & $59.6 \%(2.4)$ & $30.7 \%(2.2)$ & $90.3 \%(2.3)$ & $22.1 \%(2.4)$ \\
AD & $53.5 \%(2.7)$ & $36.1 \%(2.5)$ & $89.6 \%(2.7)$ & $22.4 \%(2.3)$ \\
\hline
\end{tabular}




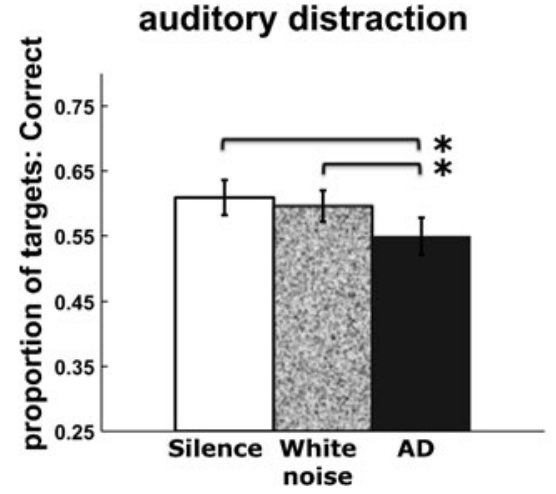

Fig. 2 Impact of distraction on episodic retrieval. The mean conditionally correct score is shown across conditions for the experiments with auditory distraction and visual distraction (Wais et al., 2010). When episodic retrieval of target images was tested with written cues, recollection was disrupted in the auditory distraction (AD) condition, relative to silence and white noise. A prior experiment

(distractor modality: auditory, visual) $\times 3$ (condition: no distractor, control distractor, distractor) ANOVA of conditional correct scores. The results showed a main effect of condition, $F(2,104)=8.68, p<.001$, such that retrieval of relevant visual details during the distractor condition declined relative to both the no-distractor and control distractor conditions (pairwise comparisons: $p<.001$ and $p<.02$, respectively). There was no difference in the pairwise comparison between the no-distractor and control distractor conditions. Critically, there was no main effect of distractor modality, $F(1,52)=0.41$, and no interaction between condition and distractor modality, $F(2,104)=1.88$, $p=.16$. Thus, the comparison across the experiments revealed that there was no main effect of distractor modality and showed that auditory distraction and visual distraction induced equivalent interference effects on recollection of task-relevant visual details.

\section{Discussion}

Recent research has revealed that the presence of irrelevant visual information has a negative impact on the recollection of visual details (Wais et al., 2010). The present results complement this finding by showing an equivalent impact of irrelevant auditory information on the recollection of visual details. This convergence of findings leads us to conclude that the influence of distraction on retrieval of visual details is independent of the sensory domain of the distractor. Therefore, the results offer evidence that disruption of episodic retrieval by environmental distractions is the result of interference with domain-general cognitive control processes.

An important premise in our interpretation of the present results is that the impact of auditory distraction is on the retrieval of visual details, which are generated by mental visual distraction

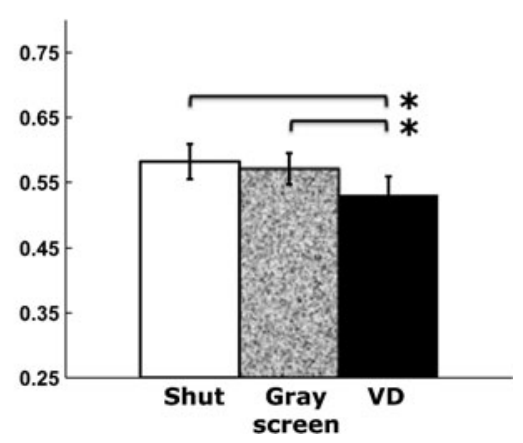

tested retrieval of the same target images with auditory cues and showed that recollection was disrupted in the visual distraction (VD) condition, relative to eyes shut and a gray screen. Error bars represent the standard error of the mean. ${ }^{* *} p<.001 ;{ }^{*} p<.05$. The level of chance for recollecting the correct count was $25 \%$

imagery processes that support the memory task. Several aspects of the study support this premise. (1) The incidental encoding tasks required visuospatial processing and were specifically designed not to encourage counting or verballabeling strategies. (2) Participants were naïve as to the memory test and had no reason to explicitly remember the number of items in, or verbal labels for, the target images. (3) Participants were specifically instructed to recall the target image cued by each probe. (4) All participants completed a postexperiment questionnaire that assessed strategies used during the memory test, and all 27 participants reported that they had used visual imagery of the target images on the majority of test trials, if not on every trial. This consistent strategy is the same as that reported by participants who completed the visual distraction experiment, as was noted in those results (Wais et al., 2010). (5) The fMRI data from Wais et al. (2010) revealed that during recall without distraction (eyes closed), participants activated the stimulus-selective visual cortex in a manner that suggests visual imagery for the targets. Therefore, we conclude that participants relied on visual imagery for target images during the memory task.

The novel and important finding of the present study that auditory distraction interferes with retrieval of episodic details suggests interesting parallels with the ISE literature examining the impact of irrelevant sound on information held in WM. Effects of auditory distraction have been previously shown to disrupt optimal WM performance (Chein \& Fiez, 2010), as well as performance on counting tasks (Buchner, Steffens, Irmen, \& Wender, 1998; Logie \& Baddeley, 1987) and tests of immediate or serial recall (Campbell et al., 2002; Marsh, Hughes, \& Jones, 2009; Salame \& Baddeley, 1982; Tremblay et al., 2000). Therefore, susceptibility to the negative impact of auditory distraction is common to both maintenance of WM and 
retrieval of visual details from LTM. Although WM and LTM are generally considered to engage dissociable cognitive domains that depend on different networks in the brain (Jeneson, Mauldin, \& Squire, 2010), results showing the comparable impact of auditory distractors suggest overlapping processes across these operations. For example, organizing lists of items in WM enhances the LTM formation of associations between those items, and increased activity in the prefrontal cortex (PFC) regions associated with WM processes predicts subsequent LTM for interitem associations (Blumenfeld \& Ranganath, 2006). Further research is required to examine the impact of distraction on processes that support both LTM retrieval and maintenance of relevant information.

The results of the present study revealed both similarities and differences with the ISE literature on the impact of white noise and more complex auditory stimuli (auditory distraction). The absence of an effect of white noise on episodic retrieval, as found in our study, is comparable to the results of studies on immediate serial recall. Notably, the impact of the ISE is absent for unaware sign-wave speech and increases from meaningless speech sounds to natural, intelligible speech (Tremblay et al., 2000), a finding that is similar to the impact of café sounds in the AD condition (i.e., a mixture of ambient restaurant noise and a few decipherable spoken words). An interesting difference is that in the present study, auditory distraction did not negatively impact overall recognition (i.e., AD did not affect $d^{\prime}$ ), yet irrelevant speech diminishes performance in studies of immediate serial recall (Beaman, 2005; Marsh et al., 2009). Moreover, white noise increased mistaken recognition of lures (i.e., false alarms) in the present study but has had no such effect on immediate serial recall (Little et al., 2010; Weisz \& Schlittmeier, 2006). These differences emphasize that the impact of irrelevant sound varies according to the memory processes engaged by particular tasks.

The convergence of results between this study, which showed effects of auditory distraction, and the previous results that revealed effects of visual distraction suggests an impact of external distraction on domain-general processes. This disruption could be explained by either top-down or bottom-up interference (although these are not mutually exclusive). For example, visual cortical regions might be impacted by bottomup influences from the multisensory processing of visual or auditory stimuli (Ghazanfar \& Schroeder, 2006). Therefore, a potential neural basis for the distraction effect is that during the $\mathrm{AD}$ condition, there is an interaction between early auditory cortical regions engaged by auditory distractors and regions in the visual association cortex that support visual imagery involved in recollection. Although the extent to which primary sensory areas in the human cortex have multisensory receptive fields is not yet known, evidence from neuroimaging implicates tuning of visual perception areas by concurrent, discordant auditory stimuli (Driver \& Noesselt, 2008; Ghazanfar \& Schroeder, 2006). Therefore, it is possible that during the $\mathrm{AD}$ condition, functional connectivity between auditory and visual regions via corticothalamic pathways may have resulted in interference on visual cortical regions engaged by memory retrieval. This interpretation suggests that because visual imagery in support of recollection utilizes the same limited-capacity buffers in the visual association cortex that are involved in processing external visual stimuli (De Fockert, Rees, Frith, \& Lavie, 2001), the fidelity of internal representations of memoranda may have been degraded by a bottom-up influence from auditory distractors.

A growing literature suggests, however, that visual imagery in the service of detailed memories is not an intrinsic phenomenon of visual cortices but is, instead, mediated by a distributed network of brain regions (Johnson et al., 2009; Mechelli, Price, Friston, \& Ishai, 2004; Wais et al., 2010). This network involves regions in the PFC, the memory regions of the medial temporal lobe, and the visual association cortex. Therefore, an alternative basis of the distraction effect on recollection is interference at a critical locus of domain-general, top-down control processes that guide selection of mnemonic details. Domain-general processes combine parallel inputs from different streams of information (i.e., representations from ventral and dorsal streams, or modality-sensitive buffers such the visuospatial sketch pad and phonological loop [Baddeley, 2010]). A parsimonious interpretation of the present results is that auditory and visual distraction produced the same impairment of episodic retrieval because both streams of irrelevant external information engaged a central cognitive control process to resolve the interference of perceptual representations on the selection of internal representations retrieved from LTM (i.e., mnemonic selection). Mnemonic selection is the process of selecting details relevant to memory retrieval goals over irrelevant competitors (Anderson \& Spellman, 1995; Badre, Poldrack, PareBlagoev, Insler, \& Wagner, 2005; Moss, Abdallah, Fletcher, Bright, Pilgrim, Acres, \& Tyler, 2005). This interpretation suggests that capacity-constrained PFC resources for topdown control may be overwhelmed if processes recruited to resolve interference from irrelevant perceptual information (Braver, Gray, \& Burgess, 2007; Gisselgard, Petersson, \& Ingvar, 2004; Nelson, Reuter-Lorenz, Persson, Sylvester, \& Jonides, 2009) or processes that incidentally mediate attention toward visual distractors (Wais et al., 2010) compete with processes that subserve visual imagery during successful recollection (Dobbins \& Wagner, 2005).

There is growing evidence from fMRI results that a particular region of the PFC is common to neural networks 
supporting both retrieval of LTM and processes engaged during interaction with the external environment during retrieval (Nee \& Jonides, 2009; Velanova, Jacoby, Wheeler, McAvoy, Petersen, \& Buckner, 2003). Wais et al. (2010) revealed that the influence of visual distraction interfered with functional connectivity in the neural network involving a ventrolateral PFC region (VLPFC, approximately BA 45), the hippocampus, and the visual association cortex, which supported recollection with eyes closed. Moreover, recent results showed that distractibility during episodic retrieval is exacerbated by repetitive transcranial magnetic stimulation (rTMS) perturbation of the left VLPFC (Wais et al., 2011). This VLPFC region has been shown to serve a critical role in the selection of contextual details during episodic retrieval (Dobbins \& Wagner, 2005), sustainment of an attentional set during retrieval mode (Velanova et al., 2003), modulation of retroactive interference from irrelevant perceptual information (Braver et al., 2007; Braver, Paxton, Locke, \& Barch, 2009), suppression of task-irrelevant actions (Chong, Williams, Cunnington, \& Mattingly, 2008), and resolution of interference during semantic retrieval (Nelson et al., 2009). Thus, our main interpretation of the present results is that an external distractor, whether within or across sensory domains, generates interference between (1) resource-limited, top-down mechanisms that guide the selection of mnemonic details available via mental imagery and (2) control processes that mediate our interactions with external distractors.

Another possibility for the basis of LTM disruption by auditory distraction is that due to limited attentional resources (Pashler \& Shiu, 1999), simultaneous, divided attention to visual and auditory stimuli (i.e., visual test cues and auditory distractors) may diminish perception of the memory cues and is reflected in reduced LTM performance (Fernandes \& Moscovitch, 2000; Talsma, Doty, \& Woldorff, 2007; Troyer, Winocur, Craik, \& Moscovitch, 1999). Recent results show, however, that division of attention between overlapping perceptual and memory tasks slows responses but does not diminish accuracy in simple recognition (Green, Johnston, \& Ruthruff, 2011). Moreover, if the present results reflected a cost of dividing attention between visual and auditory stimuli, the allocation of attention would not be expected to produce a selective effect on the accuracy of recollection for visual details. It is also important to note that participants were instructed to direct their full attention to the memory retrieval task and had no top-down goals to attend to distracting stimuli.

Further research using neuroimaging may be informative concerning the role that multisensory, bottom-up interference has on episodic retrieval, as well as elucidating mechanisms of interference with functional networks between sensory regions and PFC sources of domaingeneral top-down control.
Acknowledgment This project was supported by National Institutes of Health Grant R01-AG30395.

\section{References}

Anderson, M., \& Spellman, B. (1995). On the status of inhibitory mechanisms in cognition: Memory retrieval as a model case. Psychological Review, 102, 68-100.

Atkinson, R., \& Juola, J. (1973). Factors influencing the speed and accuracy of word recognition. In S. Kornblum (Ed.), Attention and performance IV (pp. 583-612). New York: Academic Press.

Baddeley, A. (2010). Working memory. Current Biology, 20, 136 140.

Badre, D., Poldrack, R., Paré-Blagoev, E., Insler, R., \& Wagner, A. (2005). Dissociable controlled retrieval and generalized selection mechanisms in ventrolateral prefrontal cortex. Neuron, 47, 907918.

Bakker, A., Kirwan, C. B., Miller, M., \& Stark, C. (2008). Pattern separation in the human hippocampus CA3 and dentate gyrus. Science, 319, 1640-1642.

Beaman, C. (2005). Auditory distraction from low-intensity noise: A review o the consequences for learning and workplace environments. Applied Cognitive Psychology, 19, 1041-1064.

Blumenfeld, R., \& Ranganath, C. (2006). Dorsolateral prefrontal cortex promotes long-term memory formation through its role in working memory organization. Journal of Neuroscience, 26, 916-925.

Braver, T., Gray, J., \& Burgess, G. (2007). Explaining the many varieties of working memory variation: Dual mechanisms of cognitive control. In A. Conway, C. Jarrold, M. Kane, A. Miyake, \& J. Towse (Eds.), Variation in working memory (pp. 76-106). New York: Oxford University Press.

Braver, T., Paxton, J., Locke, H., \& Barch, D. (2009). Flexible neural mechanisms of cognitive control within human prefrontal cortex. Proceedings of the National Academy of Sciences, 106, 73517356.

Buchner, A., Steffens, M. C., Irmen, L., \& Wender, K. F. (1998). Irrelevant auditory material affects counting. Journal of Experimental Psychology. Learning, Memory, and Cognition, 24, 4867.

Campbell, T., Beaman, P., \& Berry, D. (2002). Auditory memory and the irrelevant sound effect: Further evidence for changing-state disruption. Memory, 10, 199-214.

Chein, J., \& Fiez, J. (2010). Evaluating models of working memory through the effects of concurrent irrelevant information. Journal of Experimental Psychology. General, 139, 117-137.

Chong, T., Williams, M., Cunnington, R., \& Mattingly, J. (2008). Selective attention modulates inferior frontal gyrus activity during activity observation. NeuroImage, 40, 298-307.

De Fockert, J. W., Rees, G., Frith, C. D., \& Lavie, N. (2001). The role of working memory in visual selective attention. Science, 291, 1803-1806.

Dobbins, I., \& Wagner, A. (2005). Domain-general and domainsensitive prefrontal mechanisms for recollecting events and detecting novelty. Cerebral Cortex, 15, 1768-1778.

Driver, J., \& Noesselt, T. (2008). Multisensory interplay reveals crossmodal influences on 'sensory-specific' brain regions, neural responses, and judgments. Neuron, 57, 11-23.

Fernandes, M., \& Moscovitch, M. (2000). Divided attention and memory: Evidence of substantial interference effects at retrieval and encoding. Journal of Experimental Psychology. General, $129,155-176$

Ghazanfar, A., \& Schroeder, C. (2006). Is neocortex essentially multisensory? Trends in Cognitive Sciences, 10, 278-285. 
Gisselgard, J., Petersson, K., \& Ingvar, M. (2004). The irrelevant speech effect and working memory load. NeuroImage, 22, 11071116.

Green, C., Johnston, J., \& Ruthruff, E. (2011). Attentional limits in memory retrieval-revisited. Journal of Experimental Psychology. Human Perception and Performance, 37, 1083-1098.

Ishai, A., Ungerleider, L., \& Haxby, J. (2000). Distributed neural systems for the generation of visual images. Neuron, 28, 979-990.

James, W. (1890). The principles of psychology. New York: Henry Holt.

Jeneson, A., Mauldin, K., \& Squire, L. (2010). Intact working memory for relational information after medial temporal lobe damage. Journal of Neuroscience, 30, 13624-13629.

Johnson, J., \& Rugg, M. (2007). Recollection and the reinstatement of encoding-related cortical activity. Cerebral Cortex, 17, 2507-2515.

Johnson, J., McDuff, S., Rugg, M., \& Norman, K. (2009). Recollection, familiarity, and cortical reinstatement: A multivoxel pattern analysis. Neuron, 63, 697-708.

Kosslyn, S., Ganis, G., \& Thompson, W. (2001). Neural foundations of imagery. Nature Reviews Neuroscience, 2, 635-642.

Little, J., Martin, F., \& Thomson, R. (2010). Speech versus nonspeech as irrelevant sound: Controlling acoustic variation. Biological Psychology, 85, 62-70.

Logie, R. H., \& Baddeley, A. D. (1987). Cognitive processes in counting. Journal of Experimental Psychology. Learning, Memory, and Cognition, 13, 310-326.

Macmillan, N., \& Creelman, C. (2005). Detection theory: A user's guide (2nd ed.). Mahwah, NJ: Erlbaum.

Mandler, G. (1980). Recognizing: The judgment of previous occurrence. Psychological Review, 87, 252-271.

Marsh, J., Hughes, R., \& Jones, D. (2009). Interference by process, not content, determines semantic auditory distraction. Cognition, 110, 23-38.

Mechelli, A., Price, C., Friston, K., \& Ishai, A. (2004). Where bottom-up meets top-down: Neuronal interactions during perception and imagery. Cerebral Cortex, 14, 1256-1265.

Moscovitch, M. (2000). Theories of memory and consciousness. In E. Tulving \& F. Craik (Eds.), The Oxford handbook of memory (pp. 609-626). New York: Oxford University Press.

Moss, H., Abdallah, S., Fletcher, P., Bright, P., Pilgrim, L., Acres, K., et al. (2005). Selecting among competing alternatives: Selection and retrieval in the left inferior frontal gyrus. Cerebral Cortex, $15,1723-1735$.
Nee, D., \& Jonides, J. (2009). Common and distinct neural correlates of perceptual and memorial selection. NeuroImage, 25, 963-975.

Nelson, J., Reuter-Lorenz, P., Persson, J., Sylvester, C., \& Jonides, J. (2009). Mapping interference resolution across task domains: A shared control process in left inferior frontal gyrus. Brain Research, 1256, 92-100.

Pashler, H., \& Shiu, L. (1999). Do images involuntarily trigger search? A test of Pillsbury's hypothesis. Psychonomic Bulletin \& Review, 6, 445-448.

Salame, P., \& Baddeley, A. (1982). Disruption of short-term memory by unattended speech: Implications for the structure of working memory. Journal of Verbal Learning and Verbal Behavior, 21, $150-164$.

Talsma, D., Doty, T., \& Woldorff, M. (2007). Selective attention and audiovisual integration: Is attending to both modalities a prerequisite for early integration? Cerebra; Cortex, 17, 679-690.

Tremblay, S., Nicholls, A., Alford, D., \& Jones, D. (2000). The irrelevant sound effect: Does speech play a special role? Journal of Experimental Psychology. Learning, Memory, and Cognition, $26,1750-1754$.

Troyer, A., Winocur, G., Craik, F., \& Moscovitch, M. (1999). Source memory and divided attention: Reciprocal costs to primary and secondary tasks. Neuropsychology, 13, 467-474.

Tulving, E. (1985). Memory and consciousness. Canadian Journal of Psychology, 26, 1-12.

Velanova, K., Jacoby, L., Wheeler, M., McAvoy, M., Petersen, S., \& Buckner, R. (2003). Functional-anatomic correlates of sustained and transient processing components engaged during controlled retrieval. Journal of Neuroscience, 23, 8460-8470.

Wais, P., Kim, O., \& Gazzaley, A. (2011). Distractibility during episodic retrieval is exacerbated by perturbation of left ventrolateral prefrontal cortex. Cerebral Cortex. doi:10:1093/cercor/bhr160

Wais, P., Rubens, M., Boccanfuso, J., \& Gazzaley, A. (2010). Neural mechanisms underlying the impact of visual distraction on retrieval of long-term memory. Journal of Neuroscience, 30, $8541-8550$.

Weisz, N., \& Schlittmeier, S. (2006). Detrimental effects of irrelevant speech on serial recall of visual items are reflected in reduced visual N1 and reduced theta activity. Cerebral Cortex, 16, 10971105.

Wheeler, M., \& Buckner, R. (2004). Functional-anatomic correlates of remembering and knowing. NeuroImage, 21, 1337-1349. 